\title{
Multitrace matrix models of fuzzy field theories
}

\author{
Mária Šubjaková*, Juraj Tekel \\ Department of Theoretical Physics \\ Faculty of mathematics, physics and informatics \\ Comenius University, Bratislava, Slovakia \\ E-mail: maria.subjakova@fmph.uniba.sk, juraj.tekel@fmph.uniba.sk
}

We review analytical approaches to scalar field theory on fuzzy spaces. We briefly outline the matrix description of these theories and describe various approximations to the relevant matrix model. We discuss the challenge of obtaining a consistent approximation that includes the higher moments of the theory.

Corfu Summer Institute 2019 "School and Workshops on Elementary Particle Physics and Gravity" (CORFU2019)

31 August - 25 September 2019

Corfù, Greece

${ }^{*}$ Speaker. 


\section{Introduction}

Field theories defined on fuzzy spaces provide an important setting to the study of the consequences of non-commutativity of the underlining space. With their finite degrees of freedom, fuzzy spaces are readily accessible by numerical computation tools. Therefore, they have been extensively studied by these methods $[1,2,3,4,5,6,7,8]$, showing very different properties than their commutative counterparts even in the commutative limit.

Fuzzy field theories have been studied also analytically $[9,10,11,12,13,14,15,16,17,18$, 19, 20, 21]. However, the corresponding matrix models are complicated, and their full solution has not been obtained yet. Therefore, various approximations of the model have been considered.

In this paper, we review such analytical approaches mainly for the fuzzy sphere. However, the same analysis can be used for other fuzzy spaces, e.g. higher fuzzy $\mathbb{C} P^{n}$ spaces.

We start with a brief overview of the matrix model corresponding to the scalar field theory on the fuzzy sphere. Afterwards, we discuss the non-perturbative second model approximation of the model and compare it to the numerical simulations of the full model. In the last section, we review the perturbative approximation that considers also few higher moments of the theory, and demonstrate the importance of inclusion of these higher moments in a non-perturbative way.

\section{Scalar fuzzy field theories}

Fuzzy spaces are compact non-commutative spaces. The algebra of functions defined on such spaces has a finite number of degrees of freedom and can be defined as some matrix algebra [22, 23, 24, 26].

Scalar field theories on fuzzy spaces can be given in terms of the correlation functions:

$$
\langle O[M]\rangle=\frac{1}{Z} \int d M e^{-S[M]} O[M]
$$

and therefore correspond to random matrix models with probability measure given by the action of the theory.

One of the simplest example of fuzzy spaces is fuzzy sphere [25]. The algebra of functions on the fuzzy sphere is spawned by the $N \times N$ matrices:

$$
X_{i}=\frac{2 R}{\sqrt{N^{2}-1}} L_{i},
$$

where $L_{i}$ are $s u(2)$ algebra generators and $R$ gives the sphere radius. It is straightforward to check that these matrices satisfy the non-commutative relation:

$$
\left[X_{i}, X_{j}\right]=i \frac{2 R}{\sqrt{N^{2}-1}} \varepsilon_{i j k} X_{k}, \quad \sum_{i=1}^{3} X_{i} X_{i}=R^{2} .
$$

We can verify that the large $N$ limit corresponds to the commutative limit as we recover algebra of the ordinary commutative sphere functions in (2.3).

The real scalar field theory on the fuzzy sphere is given as hermitian random matrix model (2.1) with the action:

$$
S[M]=\operatorname{Tr}\left(\frac{1}{2} m^{2} M^{2}+g M^{4}+\frac{1}{2} M \mathscr{K} M\right),
$$


The kinetic term in the action (2.4) corresponds to the $s u(2)$ quadratic Casimir operator:

$$
\mathscr{K} M=\left[L_{i},\left[L_{i}, M\right]\right] .
$$

We consider only the quartic theory with the interaction parameter $g$.

To treat such models analytically in the large $N$ limit we need to rewrite the integral (2.1) in terms of different integration parameters- the eigenvalues of the matrices and the remaining angular degrees of freedoms. This leads to a model:

$$
Z=\int\left(\prod_{i=1}^{N} d \lambda_{i}\right) e^{-N^{2}\left[\frac{1}{2} m^{2} \frac{1}{N} \sum \lambda_{i}^{2}+g_{N} \frac{1}{N} \sum \lambda_{i}^{4}-\frac{2}{N^{2}} \sum_{i<j} \log \left|\lambda_{i}-\lambda_{j}\right|\right]} \int d U e^{-\frac{1}{2} \operatorname{Tr}\left[U^{\dagger} \Lambda U \mathscr{K}\left(U^{\dagger} \Lambda U\right)\right]}
$$

where $\lambda_{i}$ denotes the matrix eigenvalues, $\Lambda=\operatorname{diag}\left(\lambda_{1}, \lambda_{2}, \ldots, \lambda_{N}\right)$ and $U \in U(N)$.

The kinetic term is not invariant under unitary transformations. Therefore the angular integral in (2.6) is non-trivial and we are unable to perform the integration analytically. Thus the kinetic contribution in the action presents a significant obstacle in the analytical attempts to obtain the model solution.

As the full analytical solution of the model (2.6) cannot be attained, the approximations of the model have been considered. In the next sections we review some of these approximative techniques.

\section{Second moment approximation}

For the following sections it is useful to define the notion of the effective action as

$$
e^{-N^{2} S_{e f f}[\Lambda]}=\int d U e^{-\frac{1}{2} \operatorname{Tr}\left[U^{\dagger} \Lambda U \mathscr{K}\left(U^{\dagger} \Lambda U\right)\right]} .
$$

The effective action depends only on the eigenvalues or equivalently, due to the kinetic term invariance under translation $M \rightarrow M+a \mathbb{I}$, on the symmetrized moments of the theory:

$$
t_{n}=\operatorname{Tr}\left(M-\frac{1}{N}(\operatorname{Tr} M) \mathbb{I}\right)^{n}
$$

The analytical solution that can be obtained in the case of the free theory, i.e. for $g=0[15,16]$, provides us with the possibility to determine a part of the effective action that depends on the second symmetrized moment non-perturbatively [17]. The effective action then can be rewritten in the form:

$$
S_{\text {eff }}=\frac{1}{2} F\left(t_{2}\right)+R
$$

where the function $F\left(t_{2}\right)$ captures the known results in the case of the free theory and the remaining part $R$ gives zero contribution for such free model. For the fuzzy sphere the second moment function comes out as:

$$
F\left(t_{2}\right)=\log \left(\frac{t_{2}}{1-e^{-t_{2}}}\right)
$$

We can then approximate the effective action with this function and drop the remaining term as an approximation. Such matrix models that depend only on the eigenvalues can be studied in 
the commutative limit using the saddle point method [19, 22, 23, 27, 28]. This method tells us that in the commutative limit $N \rightarrow \infty$, only the most probable eigenvalue distribution contributes to the integral (2.6). This is the configuration satisfying:

$$
\frac{\partial S}{\partial \lambda_{i}}=0
$$

For fuzzy field theories, this saddle point equation gives three types of solutions depending on the values of parameters in the action $m^{2}, g$. Those three types of solutions are referred to as:

- the disorder phase - where the matrix eigenvalues are distributed symmetrically around zero, over one continuous interval,

- the uniform order phase- in which the eigenvalues are distributed around one of the minima of the potential $V(\lambda)=\frac{1}{2} m^{2} \lambda^{2}+g \lambda^{4}$ for the negative values of $m^{2}$,

- the non-uniform order phase- in this case, the eigenvalue distribution is not supported over one continuous interval, but half of the eigenvalues lay around one of the potential minima and a half around the other one.

The last phase does not exist in commutative field theories, it is typical for fuzzy field theories $[9,29,30,31,32]$. This phase is non-local as it corresponds to the field oscillating around different potential minima in different parts of space and is regarded as a consequence of so-called UV/IR mixing [33, 34].

This is the scale mixing phenomenon that plagues fuzzy field theories, as one cannot separate the processes on small and large scales. The more we localise the event in one dimension, the more de-localised it became in the other ones. The UV/IR mixing does not disappear in the commutative limit. Therefore the non-uniform phase remains also present.

More than one solution may be possible for some values of the parameters $m^{2}, g$. In such case the solution with lower free energy is realized:

$$
\mathscr{F}=-\frac{1}{N^{2}} \log \left(\int d M e^{-N^{2} S[M]}\right) .
$$

The saddle point equation was solved for the fuzzy sphere numerically in [18]. The perturbative solution in large negative $m^{2}$ parameter was obtained in [20]. The fact that though the perturbative expansions itself do not converge, the Padé approximations of the series give reasonable results in agreement with the numerical solution was used. The obtained results are pictured in Figure 1.

We can see that three transition lines meet at the triple point of the theory, as is expected from the numerical simulations of the full model (2.6). The location of the triple point was determined as $g_{c}=0.0048655$. These results are in a reasonable agreement with the most recent numerical simulation [1]. Thus the second moment approximation works reasonably well around the origin of the parameter space.

However, it gives qualitatively very different results further from the origin. As we can see in Figure 1, the transition line between the uniform order and non-uniform order phase asymptotically 
approaches a finite value $g=\frac{1}{16 e^{3 / 2}}$. Therefore, the uniform order phase is not realized at all for the larger values of the interaction parameter $g$. This is in disagreement with the numerical simulations which suggest that the uniform order phase reaches all the values of $g$ and the transition line behaves linearly.

These differences are the consequence of the considered approximation, and we need to include also the higher moments of the theory in order to study the model further from the origin in the parameter space.

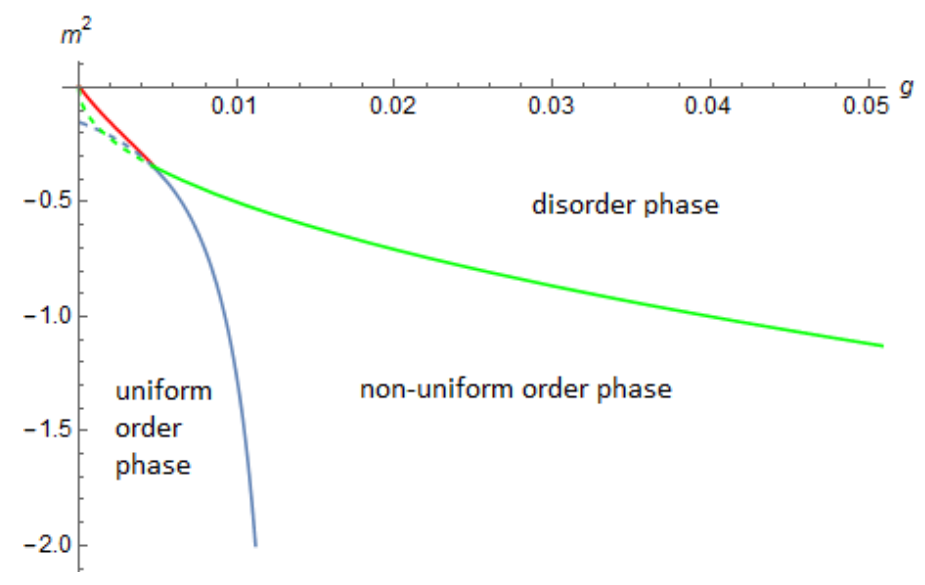

Figure 1: The phase diagram of the second moment approximation (3.4), obtained in [20]. The green line denotes the transition line between the disorder phase and the non-uniform order phase, the red line the transition between the disorder phase and the uniform order phase. The transition line between the uniform order and the non-uniform phase is pictured in blue.

\section{Beyond second moment approximation}

As we have discussed in the previous section, the second moment approximation does not capture well all of the features of the full model. Mainly, it comes short further from the origin of the parameter space. Thus, the inclusion of higher moments is needed.

An approximation that considers also higher moments in some way comes from the perturbative expansion of the unitary integral in $(2.6)[10,11]$. This expansion was obtained up to the fourth order and leads to the following multitrace expression for the effective action [12]:

$$
S_{\text {eff }}=\frac{1}{2}\left(\frac{1}{2} t_{2}-\frac{1}{24} t_{2}^{2}+\frac{1}{2880} t_{2}^{4}\right)-\frac{1}{432} t_{3}^{2}-\frac{1}{3456}\left(t_{4}-2 t_{2}^{2}\right)^{2} .
$$

Note that in the previous section, we reviewed the approximation that disregarded all higher moments completely, but the terms depending on the second moment were considered non-perturbatively. Now we include also a few higher moments, however, we only take the first terms of the perturbative expansions as the full functions are unknown.

The model with the effective action (4.1) was solved numerically in [19] and led to the phase structure shown in Figure 2.

We see that this approximation completely fails to reproduce the phase structure near the origin of the parameter phase. The phase transition lines do not intersect and there is no triple 


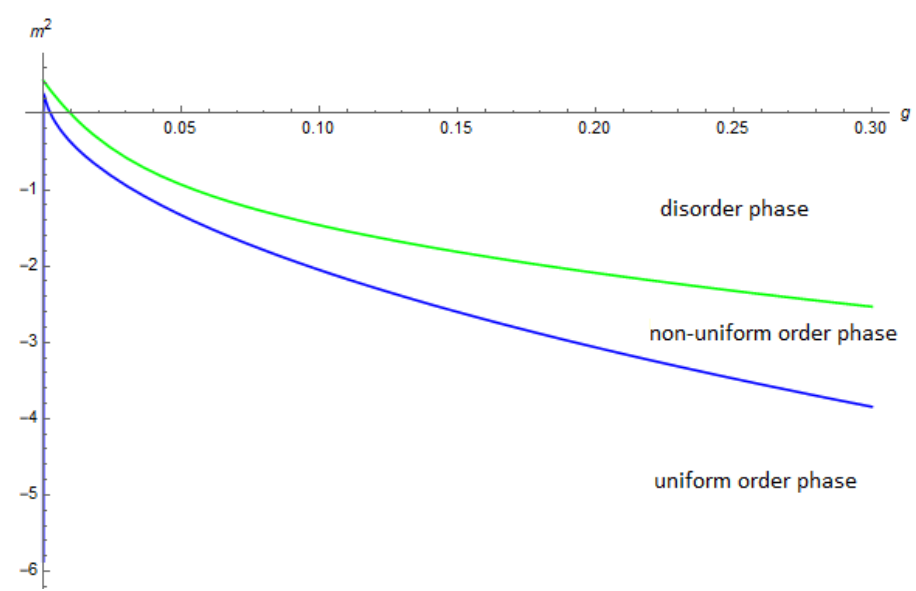

Figure 2: The phase structure of the model (4.1), obtained in [19]. The green line denotes the transition between the disorder and the non-uniform order phase, the blue line pictures the transition between the non-uniform order and uniform order phase.

point. Moreover, the transition lines do not cross the origin of the parameter space, and the phase transition between the uniform order and the non-uniform order does not behave linearly.

Despite this, the approximation has some relevance further from the origin, particularly for the disorder phase, as the moments of the solutions are small for the large interaction parameter $g$.

So we see that the multitrace expansion of the effective action is not a sufficient tool to study the phase structure of the fuzzy field theories and we need to consider higher moments nonperturbatively. These non-perturbative functions are not known, so it seems like we cannot do much more. However, it could be useful to study what kinds of changes to the phase structure we can achieve by including these higher moments non-perturbatively. We can, therefore, consider various functions of higher moments and study their effect on the phase diagram.

The higher moment functions cannot be completely arbitrary. In addition to the first known orders of their small expansions (4.1), the properties of the full model (2.6), known from the numerical simulation, present some other restrictions. For example, following functions of the higher moments could be considered:

$$
F_{3}\left(t_{3}\right)=a \log \left(1+t_{3}^{2}\right) \quad \text { or } \quad F_{3}\left(t_{3}\right)=\log \left(1+b t_{3}^{2}\right)
$$

and equivalently for the function of the fourth moment $F_{4}\left(t_{4}-2 t_{2}^{2}\right)$, with the coefficients $a, b$ set to match the multitrace expansion (4.1).

To study such general functions, analytical tools are necessary. The analytical perturbative method that was used to solve the second moment approximation model in [20] can be generalised also to the higher moments. The saddle point equations can be solved perturbatively in the large negative parameter $m^{2}$ also when considering the functions of the third and fourth moments with well behaved small and large expansions. Therefore, this method provides us with a possibility to study exactly such higher moments models.

However, this generalisation is not completely straightforward, especially for such functions of higher moments that increase at higher than the logarithmic rate for the large values of their parameters. Moreover, obtaining the transition line between the disorder phase and the uniform 
order phase is technically difficult even in the second moment approximation, due to the fact that the disorder phase does not exist in the large negative $m^{2}$ limit. In the case of the second moment approximation this problem is bypassed by expanding the solutions around the transition between the disorder and the non-uniform order phase, which is known exactly. However, for the general effective action that depends also on higher moments, this is not always the case.

Nevertheless, we believe that these problems can be handled and we are currently working on the consistent inclusion of the higher moments to the effective action.

\section{Conclusions and outlook}

We reviewed two main approaches to the analytical treatment of the matrix models corresponding to the fuzzy scalar field theories. The first approach considered only the second moment of the theory but did it in a non-perturbative way. This model gave good results near the origin of the parameter space, but further away it was inconsistent with the numerical simulations.

The second approach also considered the third and forth moments of the theory but only in a perturbative way. This model did not reproduce the phase structure of fuzzy field theory at all and, therefore, highlighted the importance of including the higher moments in a non-perturbative way.

However, these higher moments terms are known only perturbatively. Therefore, we outlined the possibility to study the general effective action depending also on these higher moments. This option arose recently with development of the analytical perturbative tools to solve the saddle point condition (3.5) for the second moment approximation. However, the road to the complete generalisation of this approach to the higher moment approximation is yet long.

\section{References}

[1] S. Kováčik, D. O'Connor, Triple Point of a Scalar Field Theory on a Fuzzy Sphere, JHEP 1810 (2018) 010 [hep-th/1805.08111].

[2] F. Garcìa Flores, X. Martin, D. O'Connor, Simulation of a scalar field on a fuzzy sphere, Int. J. Mod. Phys. A 24 (2009) 3917 [hep-lat/ 0903.1986 ].

[3] B. Ydri, New Algorithm and Phase Diagram of Noncommutative $\phi^{4}$ on the Fuzzy Sphere, JHEP 1403 (2014) 065 [hep-th/1401.1529].

[4] F. Lizzi, B. Spisso, Noncommutative Field Theory: Numerical Analysis with the Fuzzy Disc, Int. J. Mod. Phys. A 27 (2012) 1250137 [hep-th/1207 . 4998].

[5] J. Medina, W. Bietenholz, D. O'Connor, Probing the fuzzy sphere regularization in simulations of the $3 d \lambda \phi^{4}$ model, JHEP 0804 (2008) 041 [hep-th/ 0712 . 3366].

[6] H. Mejía-Díaz, W. Bietenholz, M. Panero, The Continuum Phase Diagram of the $2 d$ Non-Commutative $\lambda \phi^{4}$ Model, JHEP 1410 (2014) 056 [hep-lat/1403.3318].

[7] M. Panero, Numerical simulations of a non-commutative theory: The Scalar model on the fuzzy sphere, JHEP 0705 (2007) 082 [hep-th/0608202].

[8] B. Ydri, K. Ramda, A. Rouag, Phase diagrams of the multitrace quartic matrix models of noncommutative $\phi^{4}$ theory, Phys. Rev. D 93 (2016) 065056 [hep-th/1509. 03726]. 
[9] G. S. Gubser, S. L. Sondhi, Phase structure of non-commutative scalar field theories, Nucl. Phys. B 605 (2001) 395, [hep-th/ 0006119$].$

[10] D. O’Connor, C. Sämann, Fuzzy Scalar Field Theory as a Multitrace Matrix Model, JHEP 0708 (2007) 066 [hep-th/0706.2493].

[11] C. Sämann, The Multitrace Matrix Model of Scalar Field Theory on Fuzzy $\mathbb{C P}^{n}$, SIGMA 6 (2010) 050 [hep-th/1003.4683].

[12] C. Sämann, Bootstrapping fuzzy scalar field theory, JHEP 1504 (2015) 044 [hep-th/1412.6255].

[13] S. Rea, C. Sämann, The Phase Diagram of Scalar Field Theory on the Fuzzy Disc, JHEP 1511 (2015) 115 [hep-th/1507.05978].

[14] M. Ihl, Ch. Sachse, Ch. Sämann, Fuzzy Scalar Field Theory as Matrix Quantum Mechanics, JHEP 1103 (2011) 091, [hep-th/1306.6645].

[15] H. Steinacker, A Non-perturbative approach to non-commutative scalar field theory, JHEP $\mathbf{0 5 0 3}$ (2005) 075 [hep-th/0501174].

[16] V.P. Nair, A.P. Polychronakos, J. Tekel, Fuzzy spaces and new random matrix ensembles, Phys. Rev. D 85, (2012) 045021 [hep-th/ 0706.2493$].$

[17] A. P. Polychronakos, Effective action and phase transitions of scalar field on the fuzzy sphere, Phys. Rev. D 88 (2013) 065010 [hep-th/1306.6645].

[18] J. Tekel, Asymmetric hermitian matrix models and fuzzy field theory, Phys.Rev. D 97 (2018) 125018 [hep-th/1711.02008].

[19] J. Tekel, Phase structure of fuzzy field theories and multitrace matrix models, Acta Phys. Slov. 65 (2015) 369 [hep-th/1512.00689].

[20] M. Šubjaková, J. Tekel, Second moment fuzzy-field-theory-like matrix models, [hep-th/2002.02317].

[21] B. P. Dolan, D. O'Connor, P. Presnajder, Matrix $\phi^{4}$ models on the fuzzy sphere and their continuum limits, JHEP $\mathbf{0 2 0 3}$ (2002) 013 [hep-th/ 0109084 ].

[22] A.P. Balachandran, S. Kurkcuoglu, S. Vaidya, Lectures on Fuzzy and Fuzzy SUSY Physics, World Scientific, Singapore 2007 [hep-th/0511114].

[23] B. Ydri, Lectures on Matrix Field Theory, Springer, Cham 2017 [hep-th/1603 . 00924 ].

[24] R.J. Szabo, Quantum Field Theory on Noncommutative Spaces, Phys. Rept. 378 (2003) 207 [hep-th/0109162].

[25] J. Madore, The Fuzzy sphere, Class. Quant. Grav. 9 (1992) 69

[26] H. Grosse, C. Klimčík, P. Prešnajder, Towards Finite Quantum Field Theory in Non-Commutative Geometry, Int. J. Theor.Phys. 35 (1996) 231 [hep-th/ 9505175 ].

[27] B. Eynard, T. Kimura, S. Ribault, Random matrices, [math-ph/1510 . 04430 ].

[28] M. Šubjaková, J. Tekel, Fuzzy field theories and related matrix models, to appear in PoS CORFU2019.

[29] J. Glimm, A.M. Jaffe, T. Spencer, Phase transition for $\phi_{2}^{4}$ quantum fields, Comm. Math. Phys. 45 (1975) 203. 
[30] S. Chang, Existence of a second-order phase transition in a two-dimensional $\phi^{4}$ field theory, Phys. Rev. D 13 (2016) 2778.

[31] W. Loinaz, R.S. Willey, Monte Carlo Simulation Calculation of Critical Coupling Constant for Continuum $\phi_{2}^{4}$, Phys. Rev. D 58 (1998) 076003 [hep-lat/9712008].

[32] D. Schaich, W. Loinaz, Improved lattice measurement of the critical coupling in theory, Phys. Rev. D 79 (2009) 056008 [hep-lat/ 0902 .0045].

[33] S. Minwalla, M. Van Rammsdonk, N. Seiberg, Non-commutative perturbative dynamics, JHEP 0002 (2000) 020 [ hep-th/9912072].

[34] C.S. Chu, J. Madore, H. Steinacker, Scaling limits of the fuzzy sphere at one loop, JHEP 0108 (2001) 038 [hep-th/0106205]. 